\title{
SOME GAMMA FUNCTION INEQUALITIES
}

\author{
HORST ALZER
}

\begin{abstract}
A class of completely monotonic functions are presented involving the gamma function as well as the derivative of the psi function. As a consequence, new upper and lower bounds for the ratio $\Gamma(x+1) / \Gamma(x+s)$ are obtained and compared with related bounds given in part by J. D. Kečkić and P. M. Vasić. Our results are further applied to obtain functions which are Laplace transforms of infinitely divisible probability measures.
\end{abstract}

\section{INTRODUCTION}

In $1959 \mathrm{~W}$. Gautschi [8] presented the following remarkable inequalities for the ratio $\Gamma(n+1) / \Gamma(n+s)$ :

$$
n^{1-s}<\frac{\Gamma(n+1)}{\Gamma(n+s)}<\exp [(1-s) \psi(n+1)], \quad 0<s<1, n=1,2, \ldots,
$$

where $\psi=\Gamma^{\prime} / \Gamma$ denotes the logarithmic derivative of the gamma function. The inequalities (1.1) have found great interest, and several intriguing papers were subsequently published, for instance, by T. Erber [5], J. D. Kečkić and P. M. Vasić [10], A. Laforgia [12], and S. Zimering [17], providing new bounds for $\Gamma(n+1) / \Gamma(n+s)$.

The following sharpening of (1.1) was proved by D. Kershaw [11] in 1983:

$$
\begin{aligned}
\left(x+\frac{s}{2}\right)^{1-s} & <\frac{\Gamma(x+1)}{\Gamma(x+s)} \\
& <\exp \left[(1-s) \psi\left(x+\frac{s+1}{2}\right)\right], \quad 0<s<1, x>0,
\end{aligned}
$$

and three years later, J. Bustoz and M. E. H. Ismail [3] established a remarkable more general result. They proved that the two functions

$$
f_{1}(x)=\frac{\Gamma(x+s)}{\Gamma(x+1)} \exp \left[(1-s) \psi\left(x+\frac{s+1}{2}\right)\right], \quad 0<s<1,
$$

and

$$
f_{2}(x)=\frac{\Gamma(x+1)}{\Gamma(x+s)}\left(x+\frac{s}{2}\right)^{s-1}, \quad 0<s<1,
$$

Received by the editor October 25, 1991 and, in revised form, January 8, 1992.

1991 Mathematics Subject Classification. Primary 33B15, 26D20.

Key words and phrases. Gamma function, psi function, complete monotonicity, inequalities, identric mean, infinite divisibility, Laplace transform. 
are strictly completely monotonic on $(0, \infty)$. A function $f$ is said to be strictly completely monotonic on an interval $I \subset \mathbf{R}$ if $(-1)^{n} f^{(n)}(x)>0$ for all $x \in I$ and $n=0,1,2, \ldots$. If $(-1)^{n} f^{(n)}(x) \geq 0$ for all $x \in I$ and $n=0,1,2, \ldots$, then $f$ is called completely monotonic on $I$. Since

$$
\lim _{x \rightarrow \infty} f_{1}(x)=\lim _{x \rightarrow \infty} f_{2}(x)=1,
$$

inequalities (1.2) are immediate consequences of the fact that $f_{1}$ and $f_{2}$ are strictly decreasing on $(0, \infty)$.

Completely monotonic functions play a dominant role in areas such as numerical analysis [16], probability theory [6], and physics [4]. An interesting exposition of the main results can be found in [15, Chapter IV]. Because of "the importance of completely monotonic functions ... it may be of interest to add to the available list of such functions" $[9$, p. 1]. Hence, in the next section we introduce a new class of strictly completely monotonic functions and derive new upper and lower bounds for $\Gamma(x+1) / \Gamma(x+s)$. This is the main purpose of this paper.

Closely related bounds for $\Gamma(x+1) / \Gamma(x+s)$ were discovered by Kečkić and Vasić. In $\S 3$ we refine one of their inequalities and compare these bounds for $\Gamma(x+1) / \Gamma(x+s)$ with the ones deduced in $\S 2$. Finally, as an application, we present functions in $\S 4$ which are Laplace transforms of infinitely divisible probability measures.

\section{THE MAIN RESULTS}

The proof of Theorem 1 is based on the following easily established

Lemma. If $h^{\prime}$ is strictly completely monotonic on $(0, \infty)$, then $\exp (-h)$ is also strictly completely monotonic on $(0, \infty)$.

An extended version of the lemma can be found in [1, p. 83; 6, p. 441].

Theorem 1. The function

$$
\begin{aligned}
x \mapsto f_{\alpha}(x, s)= & \frac{\Gamma(x+s)}{\Gamma(x+1)} \frac{(x+1)^{x+1 / 2}}{(x+s)^{x+s-1 / 2}} \\
& \times \exp \left\{s-1+\frac{1}{12}\left[\psi^{\prime}(x+1+\alpha)-\psi^{\prime}(x+s+\alpha)\right]\right\} \quad(\alpha>0)
\end{aligned}
$$

is for every $s \in(0,1)$ strictly completely monotonic on $(0, \infty)$ if and only if $\alpha \geq 1 / 2$. Furthermore, the function

$$
x \mapsto 1 / f_{\beta}(x, s) \quad(\beta \geq 0)
$$

is for every $s \in(0,1)$ strictly completely monotonic on $(0, \infty)$ if and only if $\beta=0$.

Proof. First we show that the functions

$$
h_{1}(x)=\log f_{\alpha}(x, s) \quad(\alpha \geq 1 / 2) \quad \text { and } \quad h_{2}(x)=\log f_{0}(x, s)
$$

satisfy

$$
(-1)^{n}\left(-h_{1}^{\prime}(x)\right)^{(n)}>0 \text { and }(-1)^{n}\left(h_{2}^{\prime}(x)\right)^{(n)}>0
$$

for $x>0$ and $n=0,1,2, \ldots$. By the above lemma it follows that $x \mapsto$ $f_{\alpha}(x, s) \quad(\alpha \geq 1 / 2)$ and $x \mapsto 1 / f_{0}(x, s)$ are strictly completely monotonic on $(0, \infty)$. 
A simple calculation reveals

$$
\begin{aligned}
-h_{1}^{\prime}(x)= & \psi(x+1)-\psi(x+s)-\log \frac{x+1}{x+s}+\frac{1}{2}\left[\frac{1}{x+1}-\frac{1}{x+s}\right] \\
& -\frac{1}{12}\left[\psi^{\prime \prime}(x+1+\alpha)-\psi^{\prime \prime}(x+s+\alpha)\right] .
\end{aligned}
$$

Because of the integral representations

$$
\begin{gathered}
\int_{0}^{\infty} e^{-a t} d t=\frac{1}{a}, \quad a>0 \\
\int_{0}^{\infty}\left(e^{-a t}-e^{-b t}\right) \frac{d t}{t}=\log \left(\frac{b}{a}\right), \quad a>0, \quad b>0(\text { see }[7, \text { p. } 643]), \\
\psi(a)=-\gamma+\int_{0}^{\infty} \frac{e^{-t}-e^{-a t}}{1-e^{-t}} d t, \quad a>0(\text { see }[13, \text { p. 16] }),
\end{gathered}
$$

and

$$
\psi^{\prime \prime}(a)=-\int_{0}^{\infty} \frac{t^{2} e^{-a t}}{1-e^{-t}} d t, \quad a>0
$$

we obtain

$$
-h_{1}^{\prime}(x)=\int_{0}^{\infty}\left[e^{-t(x+s)}-e^{-t(x+1)}\right] p_{\alpha}(t) d t
$$

with

$$
p_{\alpha}(t)=\frac{12-t^{2} e^{-\alpha t}}{12\left(1-e^{-t}\right)}-\frac{1}{2}-\frac{1}{t}
$$

This implies

$$
(-1)^{n}\left(-h_{1}^{\prime}(x)\right)^{(n)}=\int_{0}^{\infty}\left[e^{-t(x+s)}-e^{-t(x+1)}\right] t^{n} p_{\alpha}(t) d t
$$

We now prove: $p_{\alpha}(t)>0$ for $t>0$ and $\alpha \geq 1 / 2$. Since $\alpha \mapsto p_{\alpha}(t)$ is increasing, and since $2^{k}>(k+1)(k+2) / 3$ for $k=3,4, \ldots$, we obtain

$$
\begin{aligned}
p_{\alpha}(t) & \geq p_{1 / 2}(t) \\
& =\frac{1}{2\left(e^{t}-1\right)} \sum_{k=3}^{\infty}\left[2^{k}-\frac{(k+1)(k+2)}{3}\right] k 2^{-k} \frac{t^{k+1}}{(k+2) !}>0,
\end{aligned}
$$

and we conclude that the integrand in (2.1) is positive for $t>0$. This leads to

$$
(-1)^{n}\left(-h_{1}^{\prime}(x)\right)^{(n)}>0 \text { for } x>0 \text { and } n=0,1,2, \ldots
$$

Now we prove the second part of the theorem. We have

$$
\begin{aligned}
h_{2}^{\prime}(x)= & -\psi(x+1)+\psi(x+s)-\log \frac{x+s}{x+1}+\frac{1}{2}\left[\frac{1}{x+s}-\frac{1}{x+1}\right] \\
& +\frac{1}{12}\left[\psi^{\prime \prime}(x+1)-\psi^{\prime \prime}(x+s)\right],
\end{aligned}
$$

and using the integral representations listed above, we obtain

$$
h_{2}^{\prime}(x)=\int_{0}^{\infty}\left[e^{-t(x+s)}-e^{-t(x+1)}\right] q(t) d t
$$


with

$$
q(t)=\frac{1}{2}+\frac{1}{t}-\frac{12-t^{2}}{12\left(1-e^{-t}\right)}
$$

Hence,

$$
(-1)^{n}\left(h_{2}^{\prime}(x)\right)^{(n)}=\int_{0}^{\infty}\left[e^{-t(x+s)}-e^{-t(x+1)}\right] t^{n} q(t) d t .
$$

It remains to show that $q(t)>0$ for $t>0$, or

$$
Q(t)=e^{t}\left(t^{3}-6 t+12\right)-6(t+2)>0 \text { for } t>0 \text {. }
$$

Since $Q(0)=Q^{\prime}(0)=0$ and $Q^{\prime \prime}(t)=e^{t} t^{2}(t+6)>0$ for $t>0$, we get from (2.2)

$$
(-1)^{n}\left(h_{2}^{\prime}(x)\right)^{(n)}>0 \text { for } x>0 \text { and } n=0,1,2, \ldots
$$

Let $\beta>0$; we suppose that $1 / f_{\beta}$ is strictly completely monotonic on $(0, \infty)$. Because of

$$
\lim _{x \rightarrow \infty} \frac{\Gamma(x+a)}{\Gamma(x+b)} x^{b-a}=1, \quad a>0, b>0(\text { see }[13, \text { p. 12] })
$$

and $\lim _{x \rightarrow \infty} \psi^{\prime}(x)=0$, we obtain

$$
\lim _{x \rightarrow \infty} 1 / f_{\beta}(x, s)=1 \text {. }
$$

By assumption, $1 / f_{\beta}$ is strictly decreasing; hence we have $f_{\beta}(x, s)<1$, or

$$
\begin{aligned}
(x+1)^{x+1 / 2} \exp \left\{s-1+\frac{1}{12}\left[\psi^{\prime}(x+1+\beta)-\psi^{\prime}(x+s+\beta)\right]\right\} & \\
< & \frac{\Gamma(x+1)}{\Gamma(x+s)}(x+s)^{x+s-1 / 2}
\end{aligned}
$$

for all $s \in(0,1)$ and $x>0$. If we let $s$ tend to 0 and then let $x$ tend to 0 , inequality $(2.3)$ reduces to $\exp \left(-1-\frac{1}{12} \beta^{-2}\right) \leq 0$. We assume that $f_{\alpha}$ (with $\alpha \geq 0)$ is strictly completely monotonic on $(0, \infty)$. This implies

$$
F_{x}(s)>F_{x}(1) \text { for } 0<s<1 \text { and } x>0 \text {, }
$$

with

$$
F_{x}(s)=\log \Gamma(x+s)-\left(x+s-\frac{1}{2}\right) \log (x+s)+s-\frac{1}{12} \psi^{\prime}(x+s+\alpha) .
$$

From (2.4) we conclude

$$
\begin{aligned}
\left.\frac{\partial F_{x}(s)}{\partial s}\right|_{s=1} & =\psi(x+1)-\log (x+1)+\frac{1}{2(x+1)}-\frac{1}{12} \psi^{\prime \prime}(x+1+\alpha) \\
& \leq 0 \text { for } x>0 .
\end{aligned}
$$

Setting

$$
g(a)=\int_{0}^{\infty} e^{-a t}\left[\frac{1}{e^{-t}-1}+\frac{1}{t}+\frac{1}{2}\right] d t, \quad a>0,
$$

we obtain (see [7, p. 824])

$$
g(a)=\psi(a)-\log (a)+\frac{1}{2 a}>-\frac{1}{12 a^{2}}
$$


and

$$
-\psi^{\prime \prime}(b)=\frac{1}{b^{2}}+\frac{1}{b^{3}}-g^{\prime \prime}(b), \quad b>0 .
$$

Thus, we get from (2.5)

$$
\begin{aligned}
0 & \geq(a+\alpha)^{3}\left[12 g(a)-\psi^{\prime \prime}(a+\alpha)\right] \\
& >-\frac{(a+\alpha)^{3}}{a^{2}}+a+\alpha+1-(a+\alpha)^{3} g^{\prime \prime}(a+\alpha) \\
& =(1-2 \alpha)-\frac{3 \alpha^{2}}{a}-\frac{\alpha^{3}}{a^{2}}-(a+\alpha)^{3} g^{\prime \prime}(a+\alpha) .
\end{aligned}
$$

Since $\lim _{x \rightarrow \infty} x^{3} g^{\prime \prime}(x)=0$ (see [7, p. 824]), the last inequality implies $\alpha \geq$ $1 / 2$. This completes the proof of Theorem 1 .

The functions $f_{\alpha}(\alpha \geq 1 / 2)$ and $1 / f_{0}$ are strictly decreasing and both tend to 1 as $x$ tends to $\infty$. This leads to the following bounds for $\Gamma(x+1) / \Gamma(x+s)$.

Corollary 2. The inequalities

$$
\begin{array}{r}
\frac{(x+1)^{x+1 / 2}}{(x+s)^{x+s-1 / 2}} \exp \left\{s-1+\frac{1}{12}\left[\psi^{\prime}(x+1+\beta)-\psi^{\prime}(x+s+\beta)\right]\right\} \\
<\frac{\Gamma(x+1)}{\Gamma(x+s)}<\frac{(x+1)^{x+1 / 2}}{(x+s)^{x+s-1 / 2}} \exp \left\{s-1+\frac{1}{12}\left[\psi^{\prime}(x+1+\alpha)\right.\right. \\
\left.\left.-\psi^{\prime}(x+s+\alpha)\right]\right\}
\end{array}
$$

$(0 \leq \beta<\alpha)$ are valid for all $s \in(0,1)$ and $x>0$ if and only if $\beta=0$ and $\alpha \geq 1 / 2$.

Remark. Since $\alpha \mapsto \psi^{\prime}(x+1+\alpha)-\psi^{\prime}(x+s+\alpha)(s \in(0,1), x>0)$ is strictly increasing on $(0, \infty)$, the upper bound for $\Gamma(x+1) / \Gamma(x+s)$ is best possible if $\alpha=1 / 2$.

In the Introduction we mentioned that several authors have studied inequalities for the ratio $\Gamma(x+1) / \Gamma(x+s)$. This is in particular true for the special case $s=1 / 2$. We refer to the paper of D. V. Slavic [14], which contains a summary of interesting inequalities for $\Gamma(x+1) / \Gamma(x+1 / 2)$. An application of (2.6) with $s=1 / 2$ yields:

Corollary 3. If

$$
a_{n}=\frac{3}{2}\left[1+\log \left(2 \frac{\Gamma^{2}((n+1) / 2)}{\Gamma^{2}(n / 2)} \frac{n^{n-1}}{(n+1)^{n}}\right)\right],
$$

then

$$
a_{n}<(-1)^{n+1}\left[\frac{\pi^{2}}{12}-\sum_{k=1}^{n}(-1)^{k+1} \frac{1}{k^{2}}\right]<a_{n+1}, \quad n=1,2, \ldots
$$

Proof. A direct computation reveals that the left-hand inequality of (2.7) holds for $n=1$. The other cases follow from (2.6) (with $s=1 / 2, \alpha=1 / 2, \beta=0$ ) 
and the formula

$$
\begin{aligned}
\frac{1}{4}\left[\psi^{\prime}\left(\frac{n}{2}+1\right)-\psi^{\prime}\left(\frac{n+1}{2}\right)\right] & =\sum_{k=1}^{\infty}(-1)^{k}(n+k)^{-2} \\
& =(-1)^{n+1}\left[\frac{\pi^{2}}{12}-\sum_{k=1}^{n}(-1)^{k+1} k^{-2}\right]
\end{aligned}
$$
0 .

We note that $\lim _{n \rightarrow \infty}(2 / n)^{1 / 2} \Gamma((n+1) / 2) / \Gamma(n / 2)=1$ implies $\lim _{n \rightarrow \infty} a_{n}=$

\section{The INEQUalities OF KeČKić AND VAsić}

In 1971, J. D. Kečkić and P. M. Vasić [10] published the double inequality

$$
\frac{b^{b-1}}{a^{a-1}} e^{a-b}<\frac{\Gamma(b)}{\Gamma(a)}<\frac{b^{b-1 / 2}}{a^{a-1 / 2}} e^{a-b}, \quad b>a \geq 1 .
$$

If we denote by

$$
I(a, b)=\frac{1}{e}\left(\frac{b^{b}}{a^{a}}\right)^{1 /(b-a)}, \quad a>0, b>0, a \neq b,
$$

the so-called identric mean, then inequalities (3.1) yield the following bounds for the $(b-a)$ th power of $I(a, b)$ :

$$
\left(\frac{b}{a}\right)^{1 / 2} \frac{\Gamma(b)}{\Gamma(a)}<I(a, b)^{b-a}=\left(\frac{e}{a}\right)^{a}\left(\frac{b}{e}\right)^{b}<\frac{b}{a} \frac{\Gamma(b)}{\Gamma(a)}, \quad b>a \geq 1 .
$$

The identric mean has been investigated intensively in recent years and many remarkable inequalities for $I(a, b)$ have been published by many authors (see [2, Chapter VI] and the references therein). However, we could not locate any other inequalities providing a relationship between the identric mean and the gamma function.

It is tempting to look for a refinement of (3.2). A natural question to ask is: What are the greatest number $r$ and the smallest number $s$ such that

$$
\left(\frac{b}{a}\right)^{r} \frac{\Gamma(b)}{\Gamma(a)}<I(a, b)^{b-a}<\left(\frac{a}{b}\right)^{s} \frac{\Gamma(b)}{\Gamma(a)}
$$

holds for all real numbers $b>a \geq 1$ ? We prove that $r=1 / 2$ and $s=$ $\gamma=0.5772 \ldots$ are the best possible constants. In particular, we provide a sharpening of the left-hand side of (3.1).

Theorem 4. The inequalities

$$
\left(\frac{b}{a}\right)^{r} \frac{\Gamma(b)}{\Gamma(a)}<I(a, b)^{b-a}<\left(\frac{b}{a}\right)^{s} \frac{\Gamma(b)}{\Gamma(a)}
$$

are valid for all real numbers $b>a \geq 1$ if and only if $r \leq 1 / 2$ and $s \geq \gamma$.

Proof. We assume that (3.3) holds for all $b>a \geq 1$. Setting $a=1$, we obtain

$$
r<u(b)<s
$$

for $b>1$, with

$$
u(b)=(1-b+b \log (b)-\log \Gamma(b)) / \log (b) .
$$


Since $\lim _{b \rightarrow \infty} u(b)=1 / 2$ and $\lim _{b \rightarrow 1} u(b)=\gamma$, we conclude from (3.4) that $r \leq 1 / 2$ and $s \geq \gamma$. It remains to show that the right-hand inequality of (3.3) with $s=\gamma$ is valid for all $b>a \geq 1$. We define for $b>1$

$$
v(b)=\gamma \log (b)+\log \Gamma(b)-b \log (b)+b .
$$

Differentiation yields

$$
b v^{\prime}(b)=\gamma+b[\psi(b)-\log (b)] .
$$

Putting $w(b)=b v^{\prime}(b)$, we obtain

$$
w^{\prime}(b)=\psi(b)+b \psi^{\prime}(b)-\log (b)-1 .
$$

Using the formula

$$
\psi(b)=\log (b)-\frac{1}{2 b}-\int_{0}^{\infty} \delta(t) e^{-b t} d t
$$

with $\delta(t)=1 /\left(e^{t}-1\right)-\frac{1}{t}+\frac{1}{2}($ see $[7$, p. 824]), we have

$$
w^{\prime}(b)=\int_{0}^{\infty} \delta(t)(b t-1) e^{-b t} d t .
$$

Because of

$$
(t \sinh (t / 2))^{2} \delta^{\prime}(t)=(\sinh (t / 2))^{2}-(t / 2)^{2}>0 \text { for } t>0,
$$

we conclude that $\delta$ is strictly increasing on $(0, \infty)$. Setting $\Delta(t)=(b t-1) e^{-b t}$, we get

$$
\begin{aligned}
w^{\prime}(b) & =\int_{0}^{1 / b} \delta(t) \Delta(t) d t+\int_{1 / b}^{\infty} \delta(t) \Delta(t) d t \\
& >\delta\left(\frac{1}{b}\right) \int_{0}^{1 / b} \Delta(t) d t+\delta\left(\frac{1}{b}\right) \int_{1 / b}^{\infty} \Delta(t) d t \\
& =\delta\left(\frac{1}{b}\right) \int_{0}^{\infty}(b t-1) e^{-b t} d t=0
\end{aligned}
$$

This implies $w(b)>w(1)=0$ for $b>1$. Thus, $v$ is strictly increasing on $[1, \infty)$, and we obtain $v(b)>v(a)$, which is equivalent to the second inequality of (3.3) with $s=\gamma$.

If we set $b=x+1$ and $a=x+s$, then (3.3) with $r=1 / 2$ and $s=\gamma$ yields the following inequalities, closely related to $(2.6)$,

$$
\frac{(x+1)^{x+1-\gamma}}{(x+s)^{x+s-\gamma}} e^{s-1}<\frac{\Gamma(x+1)}{\Gamma(x+s)}<\frac{(x+1)^{x+1 / 2}}{(x+s)^{x+s-1 / 2}} e^{s-1},
$$

which are valid for all real numbers $x$ and $s$ satisfying $s<1$ and $x+s \geq 1$. We note that (2.6) and (3.5) hold in different domains, so that both double inequalities might be of interest.

In what follows we compare the bounds for $\Gamma(x+1) / \Gamma(x+s)$ given in (2.6) and (3.5). Since $\psi^{\prime}$ is strictly decreasing on $(0, \infty)$, we obtain

$$
\psi^{\prime}\left(x+\frac{3}{2}\right)-\psi^{\prime}\left(x+s+\frac{1}{2}\right)<0 \text { for } x>0 \text { and } 0<s<1,
$$

which implies that the upper bound in (2.6) is an improvement over the upper bound in (3.5) for all $x>0$ and $s \in(0,1)$. In particular, we have shown that 
the right-hand inequality of (3.5) is valid in a larger domain than the sharper right-hand inequality of (2.6).

The situation with regard to the lower bound is different. An investigation reveals that in $M=\left\{(s, x) \in \mathbf{R}^{2} \mid 0<s<1, x+s \geq 1\right\}$ (the set where the lefthand inequalities of (2.6) and (3.5) hold) neither lower bound is best overall. First we prove that for every $s \in(0,1)$ there exists a number $x_{0}(s)$ such that for all $x \geq x_{0}(s)$ the lower bound in (2.6) is better than the one in (3.5). This is equivalent to

$$
12\left(\frac{1}{2}-\gamma\right) \log \frac{x+1}{x+s}<\psi^{\prime}(x+1)-\psi^{\prime}(x+s) .
$$

Because of

and

$$
\frac{1}{x}+\frac{1}{2 x^{2}}<\psi^{\prime}(x)<\frac{1}{x}+\frac{1}{2 x^{2}}+\frac{1}{6 x^{3}}, \quad x>0(\text { see }[7, \text { p. 823] }),
$$

$$
\begin{aligned}
& \psi^{\prime}(x+1)-\psi^{\prime}(x+s)-12\left(\frac{1}{2}-\gamma\right) \log \frac{x+1}{x+s} \\
& \quad>\frac{(1-s)\left[12\left(\gamma-\frac{1}{2}\right)(x+s)-1\right]}{(x+1)(x+s)}+\frac{1}{2(x+1)^{2}}-\frac{1}{2(x+s)^{2}}-\frac{1}{6(x+s)^{3}} .
\end{aligned}
$$

we obtain

$$
\log \frac{x+1}{x+s}>\frac{1-s}{1+x}, \quad x>0,0<s<1,
$$

This implies

$$
\lim _{x \rightarrow \infty} x^{2}\left[\psi^{\prime}(x+1)-\psi^{\prime}(x+s)-12\left(\frac{1}{2}-\gamma\right) \log \frac{x+1}{x+s}\right]=\infty,
$$

and hence

$$
\psi^{\prime}(x+1)-\psi^{\prime}(x+s)>12\left(\frac{1}{2}-\gamma\right) \log \frac{x+1}{x+s} \text { for } x \geq x_{0}(s) .
$$

Next we show that there exist $(s, x) \in M$ such that the opposite inequality of (3.6) holds. Since

$$
\begin{aligned}
\lim _{x \rightarrow 0} \frac{\psi^{\prime}(x+1)-\psi^{\prime}(1)}{\log (x+1)-\log (1)} & =\psi^{\prime \prime}(1)=-2.404 \ldots \\
& <-0.926 \cdots=12\left(\frac{1}{2}-\gamma\right),
\end{aligned}
$$

we conclude that there exists a number $\eta \in(0,1)$ such that

$$
12\left(\frac{1}{2}-\gamma\right) \log \frac{x+1}{x+s}>\psi^{\prime}(x+1)-\psi^{\prime}(x+s)
$$

is valid for all $x$ and $s$ with $x+s=1$ and $0<x<\eta$.

\section{INFINITELY DIVISIBLE PROBABILITY MEASURES}

In this section we present an application of Theorem 1 to probability theory. We recall that a probability measure $d \mu$ is infinitely divisible if for every natural number $n$ there exists a probability measure $d \mu_{n}$ such that

$$
d \mu=d \mu_{n} * d \mu_{n} * \cdots * d \mu_{n} \quad(n \text { times }),
$$

where $*$ denotes convolution. 
An interesting connection between infinitely divisible probability measures and completely monotonic functions is given by the following proposition:

A probability measure $d \mu$ supported on a subset of $[0, \infty)$ is infinitely divisible if and only if

$$
\int_{0}^{\infty} e^{-x t} d \mu(t)=e^{-h(x)}, \quad x \geq 0,
$$

where $h$ has a completely monotonic derivative on $(0, \infty)$ and $h(0)=0$. (See $[6$, p. 450].)

This theorem, and the results from $\S 2$, lead to

Theorem 5. Let $\varepsilon>0$ and $s \in(0,1)$. Then the functions

$$
x \mapsto f_{\alpha}(x+\varepsilon, s) / f_{\alpha}(\varepsilon, s) \quad(\alpha \geq 1 / 2)
$$

and

$$
x \mapsto f_{0}(\varepsilon, s) / f_{0}(x+\varepsilon, s)
$$

are Laplace transforms of infinitely divisible probability measures.

Related results can be found in [3, 9].

\section{ACKNOWLEDGMENT}

I thank the referee for helpful suggestions and F. Bullock for inspiring discussions.

\section{BIBLIOGRAPHY}

1. S. Bochner, Harmonic analysis and the theory of probability, Univ. of California Press, Berkeley/Los Angeles, 1960.

2. P. S. Bullen, D. S. Mitrinović, and P. M. Vasić, Means and their inequalities, Reidel, Dordrecht, 1988.

3. J. Bustoz and M. E. H. Ismail, On gamma function inequalities, Math. Comp. 47 (1986), 659-667.

4. W. A. Day, On monotonicity of the relaxation functions of viscoelastic materials, Proc. Cambridge Philos. Soc. 67 (1970), 503-508.

5. T. Erber, The gamma function inequalities of Gurland and Gautschi, Skand. Aktuarietidskr. 1960 (1961), 27-28.

6. W. Feller, An introduction to probability theory and its applications, Vol. 2, Wiley, New York, 1966.

7. G. M. Fichtenholz, Differential- und Integralrechnung. II, Deutscher Verlag Wiss., Berlin, 1979.

8. W. Gautschi, Some elementary inequalities relating to the gamma and incomplete gamma function, J. Math. Phys. 38 (1959), 77-81.

9. M. E. H. Ismail, L. Lorch, and M. Muldoon, Completely monotonic functions associated with the gamma function and its q-analogues, J. Math. Anal. Appl. 116 (1986), 1-9.

10. J. D. Kečkić and P. M. Vasić, Some inequalities for the gamma function, Publ. Inst. Math. (Beograd) (N.S.) 11 (1971), 107-114.

11. D. Kershaw, Some extensions of $W$. Gautschi's inequalities for the gamma function, Math. Comp. 41 (1983), 607-611.

12. A. Laforgia, Further inequalities for the gamma function, Math. Comp. 42 (1984), 597-600.

13. W. Magnus, F. Oberhettinger, and R. P. Soni, Formulas and theorems for the special functions of mathematical physics, Springer-Verlag, Berlin, 1966. 
14. D. V. Slavić, On inequalities for $\Gamma(x+1) / \Gamma(x+1 / 2)$, Univ. Beograd. Publ. Elektrotehn. Fak. Ser. Mat. Fiz. 498-541 (1975), 17-20.

15. D. V. Widder, The Laplace transform, Princeton Univ. Press, Princeton, NJ, 1946.

16. J. Wimp, Sequence transformations and their applications, Academic Press, New York, 1981.

17. S. Zimering, On a Mercerian theorem and its application to the equiconvergence of Cesàro and Riesz transforms, Publ. Inst. Math. (Beograd) (N.S.) 1 (1961), 83-91.

Morsbacher Strasse 10, 5220 Waldbröl, Germany 